\title{
Copper Nanoparticles Induce Oxidative Stress via the Heme Oxygenase I Signaling Pathway in vitro Studies
}

This article was published in the following Dove Press journal: International Journal of Nanomedicine

\section{Liping Zou (iD) \\ Guiping Cheng \\ Chengcheng $\mathrm{Xu}$ \\ Heyu Liu \\ Yingying Wang \\ Nianyu Li \\ Xiaorong Fan \\ Changhong Zhu \\ Wei Xia}

Institute of Reproductive Health, Tongji Medical College, Huazhong University of Science and Technology, Wuhan, People's Republic of China
Correspondence: Wei Xia Institute of Reproductive Health, Tongii Medical College, Huazhong University of Science and Technology, Wuhan, Hubei, 430030, People's Republic of China $\mathrm{Tel} / \mathrm{Fax}+86278369265 \mathrm{I}$

Email tjxiawei@hust.edu.cn
Purpose: The toxicity of copper nanoparticle (CuNP) exposure in the ovaries has attracted attention recently, but the precise molecular mechanism involved requires further investigation. We investigated the cytotoxicity of CuNPs in ovarian granulosa cells and the protective effect of heme oxygenase 1 (HO-1) against CuNP-induced damage.

Methods: Human ovarian granulosa cells (COV434) were treated with CuNPs, and cytotoxicity was evaluated using Cell Counting Kit- 8 and flow cytometry assays. Oxidative stress was identified using biochemical markers of oxidation and anti-oxidation. The protein levels of mitogen-activated protein kinase 14 (MAPK14), phospho-MAPK14, nuclear factor erythroid 2-related factor 2 (Nrf2), and HO-1 were measured by immunoblotting. Subsequently, for oxidative stress parameter detection, the cells were pre-treated with hemin to induce $\mathrm{HO}$ 1 expression prior to CuNP treatment.

Results: Exposure to CuNPs decreased cell viability and the mitochondrial membrane potential, increased the apoptosis rate, and induced oxidative stress. Furthermore, hemin pretreatment induced HO-1 expression in cells, which partially reduced the accumulation of reactive oxygen species induced by CuNPs and increased the levels of antioxidant enzymes. Conclusion: CuNPs exert cytotoxic effects on human ovarian granulosa cells by inducing oxidative stress, and may induce HO-1 expression via the MAPK14-Nrf2 signaling pathway. Moreover, HO-1 protects against oxidative stress induced by CuNPs.

Keywords: copper nanoparticles, cytotoxicity, oxidative stress, heme oxygenase 1

\section{Introduction}

Nanomaterials are artificial microparticles with maximum diameters of $100 \mathrm{~nm}$. As relatively common metal nanomaterials, copper nanoparticles (CuNPs) have excellent physical and chemical properties. ${ }^{1}$ CuNPs are currently used for drug delivery and antibacterial applications, as animal feed additives, as emerging environmental catalysts, in bioimaging, and in other modern technologies. ${ }^{2-4}$ With the rapid development and wide application of CuNPs, they have spread widely in the environment, increasing the risk of exposure of the general population. The toxicity of CuNPs to various living organisms has caused public concern. ${ }^{5,6}$ Numerous studies have shown that CuNPs have serious toxicological effects and induce severe damage to the liver, spleen, and kidney in vivo. ${ }^{7-9}$ Moreover, the brain and reproductive system are also target organs of toxicity. ${ }^{10-13}$

Small size is reported to be one of the reasons for the cytotoxic effects of nanoparticles. ${ }^{7,14-16}$ The cytotoxicity of CuNPs mainly comes from their ability 
to generate reactive oxygen species (ROS), ${ }^{17,18}$ which induce cell death. Previous studies have mainly focused on the cytotoxicity of CuNPs in the liver, ${ }^{19}$ kidney, ${ }^{20}$ and spleen. ${ }^{21}$ However, the ovary has recently been reported to be a target organ for CuNP toxicity. Our previous studies show that CuNPs can migrate to the ovaries of rats and accumulate in large quantities, thereby causing various types of damage such as ovarian injury, follicular atresia, oxidative stress, and apoptosis. ${ }^{11}$ The proliferation and secretory functions of granulosa cells are essential for germ cell development in animals. Granulosa cells nourish oocytes through gap junctions and regulate oocytes through paracrine. ${ }^{22}$ The ovaries of rats exposed to CuNPs exhibit increased lipid peroxidation and antioxidant system destruction. ${ }^{11}$ Therefore, we speculate that the cytotoxicity of CuNPs in ovarian granulosa cells may be related to oxidative stress.

Oxidative stress is caused by an imbalance in the generation and removal of oxygen free radicals, which causes the accumulation of ROS and reactive nitrogen species in tissues or cells. Excessive ROS production destroys proteins, nucleic acids, and lipids and overloads the antioxidant defenses, thus affecting their normal physiological and biochemical functions and leading to cell death. ${ }^{23,24}$ ROS production and oxidative stress are closely related to apoptosis induced by CuNPs. ${ }^{17}$ Heme oxygenase 1 (HO-1) is an adaptive protein that plays an important role in protecting against oxidation and apoptosis. $^{25}$ Recent studies have explored the molecular mechanism whereby HO-1 protects cells against oxidation during heat stress by reducing the accumulation of ROS and activating the antioxidant response. ${ }^{26}$ Studies have shown that HO-1 induction during oxidative stress is regulated by the mitogen-activated protein kinase 14 (MAPK14)/nuclear factor erythroid 2-related factor 2 (Nrf2) signaling pathway. ${ }^{27}$

In this study, we speculated that an increase in HO-1 expression levels may be involved in the anti-oxidative stress response in granulosa cells exposed to CuNPs. Cell proliferation, the apoptosis rate, changes in mitochondrial membrane potential, and oxidative stress were assessed to explore the cytotoxicity of CuNPs in granulosa cells. Oxidative stress markers, including ROS, superoxide dismutase 2 (SOD2), and glutathione peroxidase (GSH), were analyzed after $\mathrm{HO}-1$ induction to explore the cytoprotective properties of HO-1.

\section{Materials and Methods \\ Preparation of CuNPs}

CuNPs (100 nm) were purchased from Da Yu Medical Instrument Co. Ltd. (Wuhan, Hubei, China). These CuNPs were characterized in our previous study. ${ }^{11}$ Before use, the CuNPs were suspended in medium and dispersed by sonication for $25 \mathrm{~min}$ to prevent aggregation. Different concentrations of the CuNP suspension were obtained by diluting with complete medium.

\section{Cell Culture and Pharmacological Experiments}

The human ovarian granulosa tumor cell line COV434 was obtained from Procell (Wuhan, China). COV434 cells were cultured in a $37^{\circ} \mathrm{C}$ incubator with humidified air containing $5 \% \mathrm{CO}_{2}$. The complete medium consisted of Dulbecco's modified Eagle medium, 15\% fetal bovine serum (Gibco, Carlsbad, CA, USA), and 1\% penicillin and streptomycin. To induce HO-1 expression, COV434 cells were pre-treated with $20 \mu \mathrm{mol} / \mathrm{L}$ hemin (MedChemExpress, Monmouth Junction, NJ, USA), an HO-1-specific activator, for $48 \mathrm{~h}$.

\section{Cell Viability Detection}

Cell viability was determined using the Cell Counting Kit-8 (CCK-8) assay (Dojindo, Tokyo, Japan). COV434 cells in 96well plates were treated with different doses of CuNP suspension with a density of $1 \times 10^{5}$ cells per well. After incubation for 12 or $24 \mathrm{~h}$, the CCK 8 detection solution was added, and cells were incubated for another $2 \mathrm{~h}$. The absorbance value at $450 \mathrm{~nm}$ was measured using a multimode microplate reader (Synergy $^{\text {TM }}$ HTX; BioTek, Winooski, VT, USA). Relative cell viability was calculated using the following equation: $\%$ cell viability $=$ absorbance value $_{(\mathrm{CuNPs}}$ group - blank group $)$ /absorbance value (control group - blank group).

\section{Apoptosis Detection}

An FITC Annexin V and PI Apoptosis Kit (ABP Biosciences, Beltsville, MD, USA) was used to detect apoptotic cells. Briefly, COV434 cells $(2.5 \mathrm{~mL} /$ well $)$ were evenly plated in 6-well plates and then incubated with $150 \mu \mathrm{g} / \mathrm{mL} \mathrm{CuNPs}$ for $12 \mathrm{~h}$. The control group was not treated with CuNPs. After washing three times with phosphate-buffered saline, COV434 cells were resuspended in binding buffer containing annexin V-FITC and propidium iodide, and then incubated in the dark at $20-25^{\circ} \mathrm{C}$ for flow cytometry. 


\section{Mitochondrial Membrane Potential Detection}

Flow cytometry with JC-1 (Beyotime, Shanghai, China) was used to measure mitochondrial membrane potential (MMP). COV434 cells at a density of $1 \times 10^{6}$ cells per wells were evenly plated in 6-well plates and then incubated with $150 \mu \mathrm{g} / \mathrm{mL}$ CuNPs for $12 \mathrm{~h}$. Cells were then collected, resuspended in JC-1 staining reagent, and placed in an incubator for $20 \mathrm{~min}$. After washing with JC-1 buffer, the cell mixture was analyzed by flow cytometry.

\section{Oxidative Stress Parameters}

SOD activity and ROS levels were measured using commercially available kits (Nanjing Jiancheng, Jiangsu, China). Total SOD activity was determined according to the manufacturer's instructions. Intracellular ROS accumulation was measured using 2,7-dichlorofluorescein diacetate (DCFH-DA), as ROS oxidizes DCFH-DA into the green fluorescent compound dichlorofluorescein. Intracellular fluorescence was detected using a multimode microplate reader. The resulting fluorescence value was proportional to the intracellular ROS level. The levels of GSH and malondialdehyde (MDA) production and catalase (CAT) activity were quantified using commercially available kits (Solarbio, Beijing, China). The treated cells were collected, disrupted by ultrasonication, and centrifuged at $8000 \times \mathrm{g}$ at $4^{\circ} \mathrm{C}$ for $10 \mathrm{~min}$. The resulting supernatant was collected for analysis. The absorbance value of each sample was determined, and the results were calculated according to the manufacturer's instructions.

\section{Western Blotting}

Cells were fully lysed using radioimmunoprecipitation assay buffer (Servicebio, Wuhan, China) containing 2\% protease inhibitors and phosphatase inhibitors (Beyotime). Total protein samples were obtained after centrifugation. The extracted proteins were separated by $8-12 \%$ sodium dodecyl sulfatepolyacrylamide gel electrophoresis (Beyotime), and transferred to $0.45 \mu \mathrm{m}$ polyvinylidene fluoride membranes (Merck KGaA, Darmstadt, Germany). The membranes were blocked in 5\% skim milk, incubated with primary antibodies overnight, and then incubated with secondary antibodies (horseradish peroxidase-conjugated IgG; Abclonal, Wuhan, China). The following primary antibodies were used: antiphospho-MAPK14, -Keap1 and -NQO1 (Abclonal); antiMAPK14 (Santa Cruz, Dallas, TX, USA); anti-SOD2 (Cell
Signaling Technology, Beverly, MA, USA); anti-Nrf2, -HO-1, and -GAPDH (Proteintech, Wuhan, China). The immunoblot signals were detected using a Super-Sensitive Chemiluminescence Substrate Kit (Biosharp, Beijing, China). The protein bands were analyzed using a chemiluminescent gel imaging system, with Image Lab software (ChemiDoc XRS; Bio-Rad, Hercules, CA, USA).

\section{Statistical Analysis}

Statistical analysis was performed using a one-way ANOVA, followed by least significant difference post hoc tests. SPSS 23.0 software was used for these calculations (IBM, Chicago, IL, USA). Data are presented as the means $\pm \mathrm{SD}$. All experiments were repeated at least three times, and $p<0.05$ was considered statistically significant.

\section{Results \\ Cytotoxic Effect of CuNPs on COV434 Cells}

To examine the cytotoxicity of CuNPs in granulosa cells, COV434 cells were exposed to culture media containing different concentrations of CuNPs or without CuNPs (control). The results were compared with those of the control group. We found that COV434 cell viability decreased with an increasing treatment concentration and time (Figure 1A). After $12 \mathrm{~h}$ of CuNP exposure, the effective dose resulting in a $50 \%$ inhibition of COV434 cell growth was $150 \mu \mathrm{g} / \mathrm{mL}$.

\section{CuNPs Induce Apoptosis and Decrease MMP}

COV434 cell apoptosis was detected using a flow cytometer. The results from the CuNP group were compared to those from the control group. We observed a higher apoptosis rate in the CuNP group (Figure 1B). MMP, as measured by flow cytometry after JC-1-staining, decreased significantly in the CuNP group (Figure 1C).

\section{CuNPs Induce Oxidative Stress}

To investigate whether oxidative stress was involved in CuNP-induced cytotoxicity, relevant detection kits were used to measure biomarkers of oxidative stress in COV434 cells before and after CuNP exposure. As shown in Figure 2, the levels of the oxidation products ROS and MDA increased significantly, whereas the levels of antioxidant biochemical markers, including SOD, GSH, and CAT, decreased in the CuNP group. 

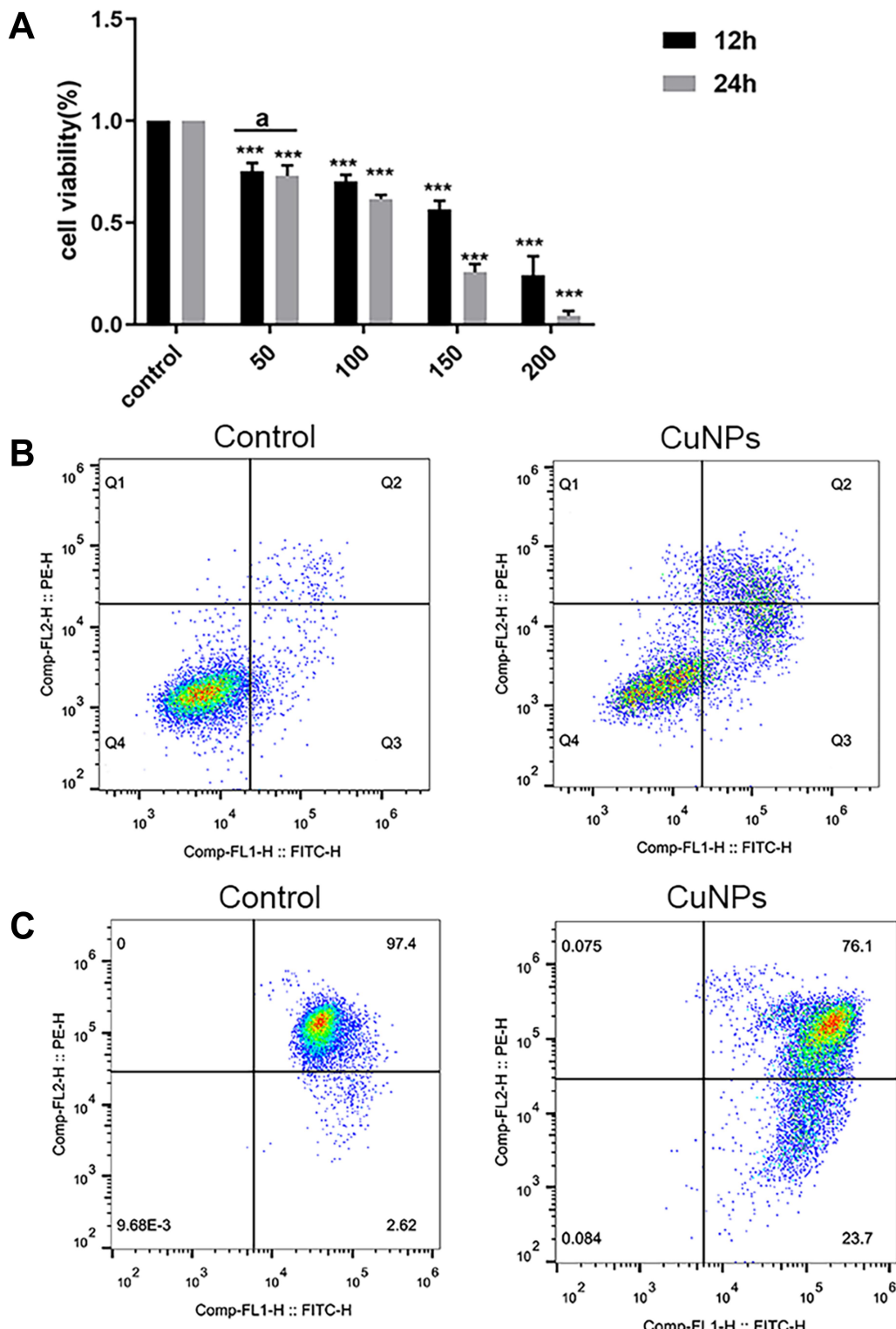
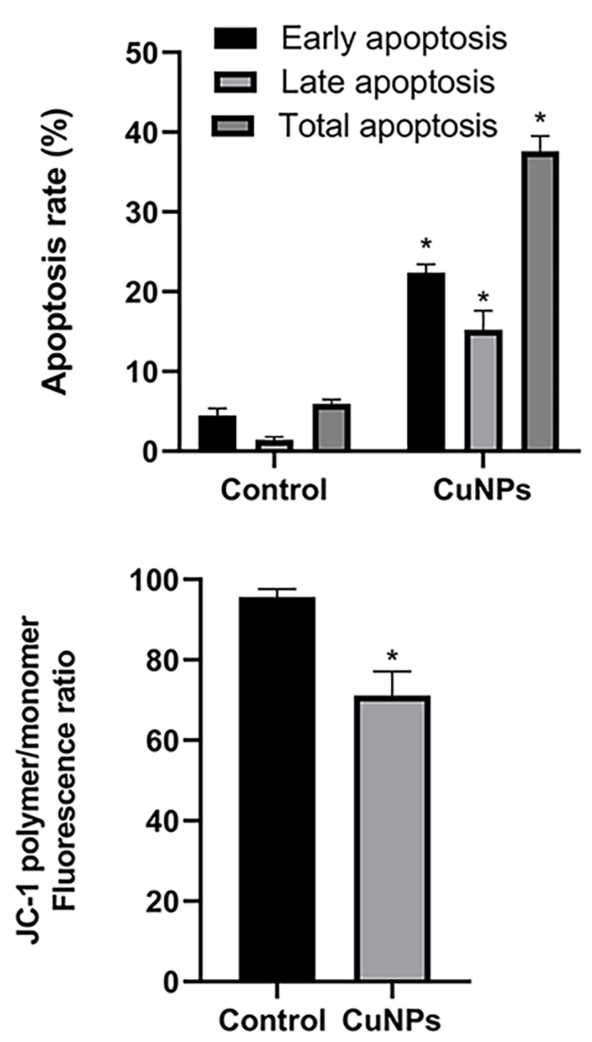

Figure I Cytotoxic effect of CuNP exposure in ovarian granulosa cells.

Notes: (A) The viability of COV434 cells after 12 and $24 \mathrm{~h}$ of treatment with different concentrations $(0,50,100,150$, and $200 \mu \mathrm{g} / \mathrm{mL})$ of CuNPs. (B) COV434 cell apoptosis before and after CuNP treatment. In the flow chart, Q3 indicates early apoptosis and Q2 indicates late apoptosis. (C) MMP in COV434 cells before and after CuNP treatment. ${ }^{a}$ Indicates no difference between groups; ${ }^{*} p<0.05$, $* * * p<0.00$ I.

Abbreviations: CuNPs, copper nanoparticles; MMP, mitochondrial membrane potential.

\section{Effect of CuNPs on the Levels of} MAPK I4, Nrf2, NAD(P)H Quinone

\section{Dehydrogenase I (NQOI), and HO-I}

To further explore the specific molecular events involved in the oxidative stress caused by CuNP exposure, members of the MAPK14 signaling pathway were analyzed by Western blotting. The expression of the unphosphorylated form of MAPK14 was constant, whereas the phosphorylation of MAPK14 was significantly induced by CuNPs (Figure 3). Meanwhile, an increase in Nrf2 expression levels and a decrease in Keap1 expression levels were also detected. HO-1 and NQO1 are encoded by Nrf2 target genes and are wellcharacterized anti-oxidant proteins. CuNPs strongly induced HO-1 expression, but had no effect on NQO1 expression. 

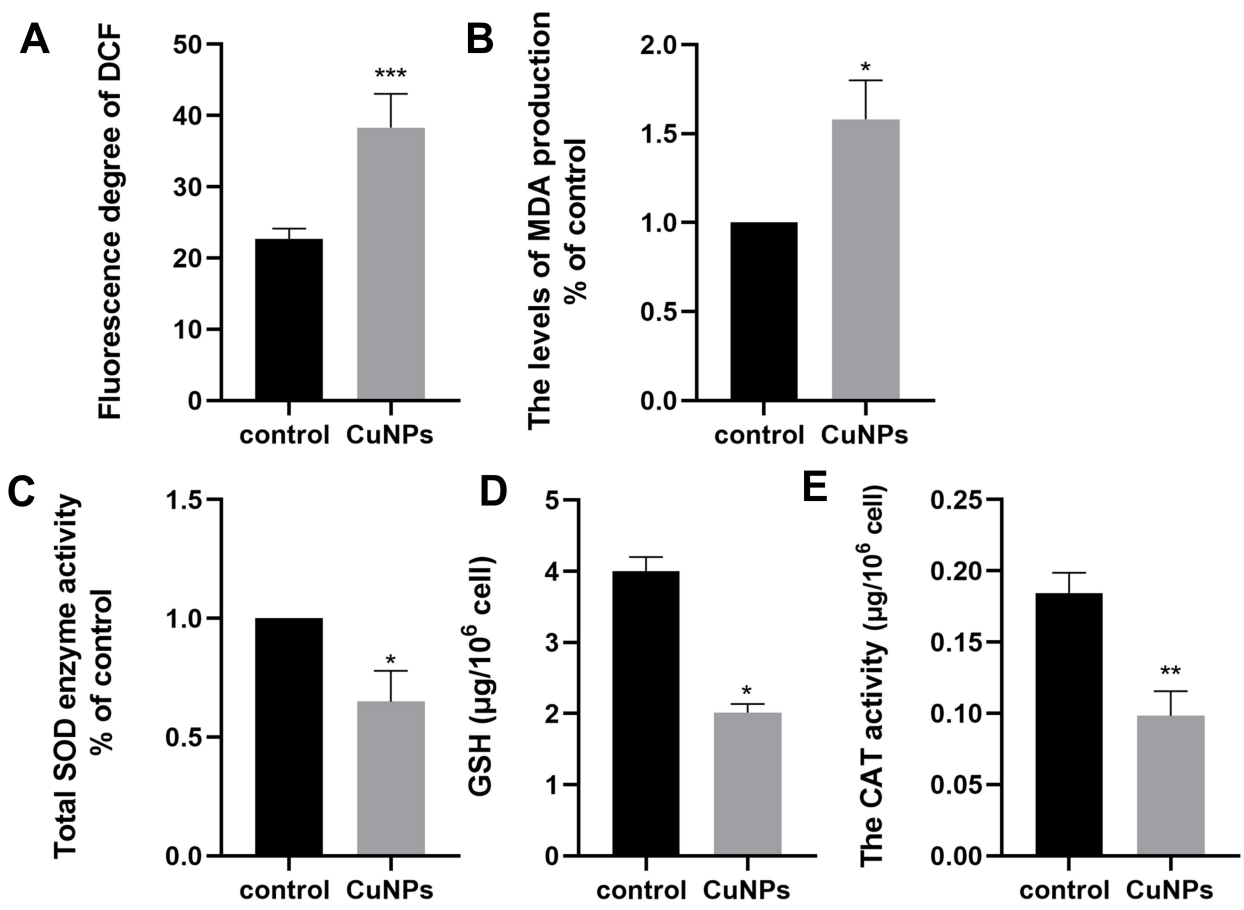

Figure 2 Effect of CuNP exposure on ROS (A), MDA (B), SOD (C), GSH (D), and CAT (E) in COV434 cells. Notes: Compared to the control group: ${ }^{*} p<0.05$, ${ }^{*} p<<0.01$, ${ }^{* * *} p<0.00$ I.

Abbreviations: ROS, reactive oxygen species; MDA, malonaldehyde; SOD, superoxide dismutase; GSH, glutathione peroxidase; CAT, catalase.

\section{HO-I Induction Attenuates ROS Generation and Increases Antioxidant Defenses in CuNP-Treated COV434 Cells}

The effect of hemin on HO-1 expression was first determined by Western blotting (Figure 4A). Based on previous reports and the results of this study, ${ }^{26,28}$ we chose 20 $\mu \mathrm{mol} / \mathrm{L}$ hemin as the optimal concentration for subsequent experiments. A significant decrease in ROS generation was detected in cells pretreated with hemin, compared to those treated with CuNPs only (Figure 4B). Meanwhile, HO-1 was induced and SOD2 protein levels were upregulated in the CuNPs+hemin group compared with the CuNPs group (Figure 4C). In addition, the GSH levels were increased in the CuNPsthemin group (Figure 4D).

\section{Discussion}

Our previous experiments demonstrated that CuNPs may adversely impact rat ovaries. ${ }^{11}$ Given limitations of animal studies, we further investigated the mechanism of CuNPinduced ovarian damage in vitro and explored possible protective mechanisms. Our results indicated that CuNPs have cytotoxic effects on COV434 cells in a dose- and time-dependent manner. We also observed an increase in the rate of apoptosis and a decrease in the MMP after
CuNP treatment. These results were consistent with those reported by Zhang et al, who showed that CuNPs can damage MMP and exert cytotoxicity in female mice via the mitochondrial apoptosis pathway. ${ }^{29}$ Oxidative stress has been associated with the mitochondria-mediated apoptotic pathway and subsequent cell death. ${ }^{1,30}$

Oxidative stress is a state of disturbance in the equilibrium between oxidative and antioxidative factors. It can be identified by analyzing various biochemical indicators that reflect the degree of destruction of the antioxidant defense system. ${ }^{31}$ To further understand the apoptosis of ovarian granulosa cells, we examined the intracellular levels of oxidative and antioxidative factors. CuNPs were found to significantly increase levels of the intracellular oxidation products, ROS and MDA, and to significantly destroy the antioxidant defenses, as indicated by reduced SOD and CAT activity and decreased GSH levels. These results suggest that the cytotoxicity of CuNPs to ovarian granulosa cells may be caused by oxidative stress.

MAPK signals are upstream of multiple stressstimulation cascade events. ${ }^{32,33}$ Oxidative damage can activate MAPK14 phosphorylation, ${ }^{21}$ which in turn promotes the separation of $\mathrm{Nrf} 2$ from Keap $1 .{ }^{27} \mathrm{Nrf} 2$ then translocates to the nucleus and binds to antioxidant response elements to activate various related genes, such 
A

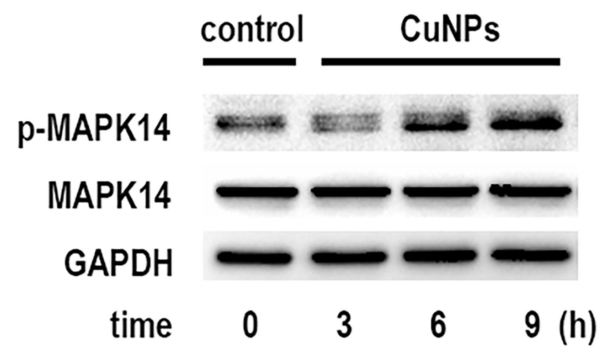

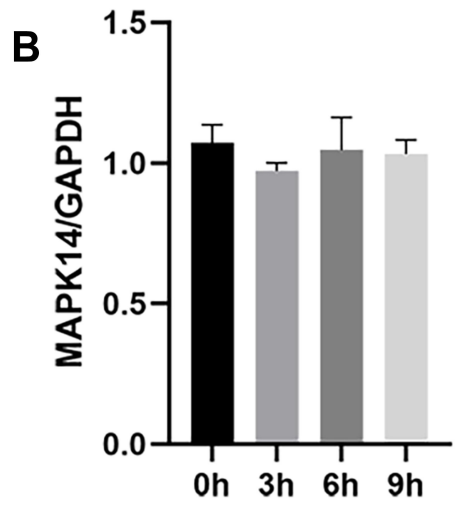

C

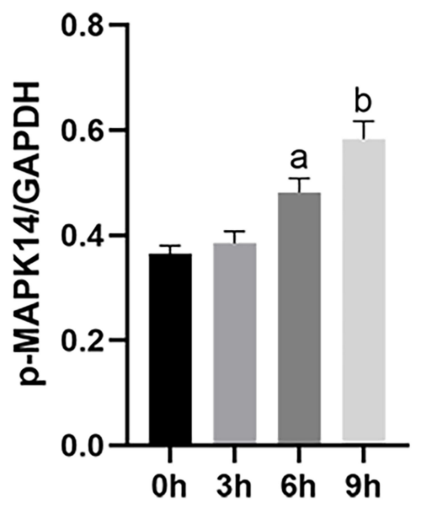

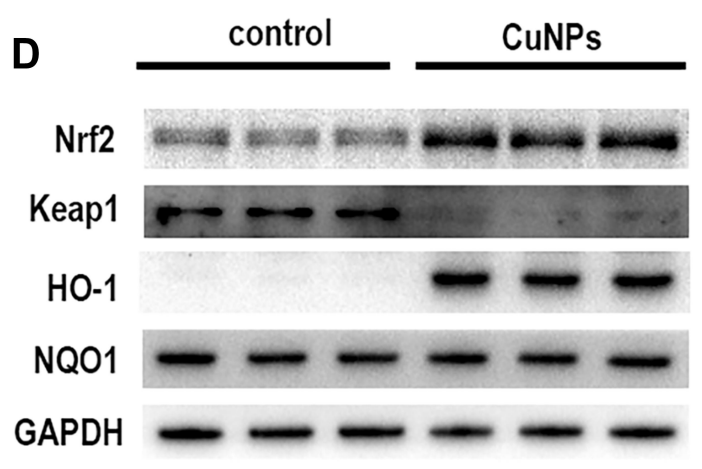

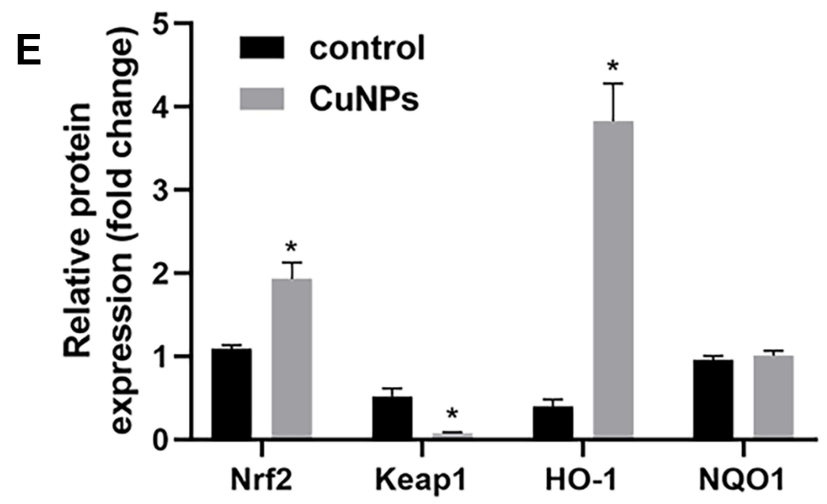

Figure 3 Levels of MAPKI4, P-MAPKI4, Nrf2, HO-I, and NQOI proteins in COV434 cells exposed to CuNPs.

Notes: (A) Western blotting images of P-MAPKI4 and MAPKI4 proteins at different times of exposure to CuNPs. (B and C) Densitometric analysis of p-MAPKI4 and MAPKI4 protein bands. (D) Western blotting images of Nrf2, Keapl, HO-I, and NQOI proteins. (E) Densitometric analysis of protein bands. * $p$ < 0.05 ; ${ }^{a}, \mathrm{~b}$ Indicate statistical significance, with the same letters indicating no difference between groups.

Abbreviations: MAPK 14, mitogen-activated protein kinase 14; Nrf2, nuclear factor erythroid 2-related factor 2; HO-I, heme oxygenase I; NQOI, NAD(P)H quinone dehydrogenase I; CuNPs, copper nanoparticles.

as $H O-1$ and $N Q O 1 .{ }^{34}$ The role of the MAPK14 signaling pathway and its related transcription factors in oxidative stress events caused by metal nanoparticles has attracted increasing attention. Some studies have demonstrated that the activation of Nrf2/HO-1 protects cells from AgNP- and $\mathrm{SiO}_{2} \mathrm{NP}$-induced oxidative stress. Moreover, $\mathrm{TiO}_{2}$ nanoparticles and naked gold nanoparticles can activate MAPK14/Nrf2/HO-1 signaling. ${ }^{35-38}$ We also detected the phosphorylation of MAPK14 and upregulation of Nrf2/ HO-1 in CuNP-treated COV434 cells. HO-1 and NQO1 are well-characterized antioxidant proteins that are regulated by activated Nrf2. CuNPs significantly induced HO-1 expression, whereas no changes in NQO1 protein levels were detected. These results may be due to the fact that we only tested protein levels. In addition, it is possible that an increase in NQO1 protein levels occurs later, or that activated Nrf2 does not regulate $N Q O 1$ transcription in this model.

HO- 1 protein is readily induced by heme, heat stress, hyperoxemia, heavy metals, ultraviolet rays, and other stimuli. ${ }^{39} \mathrm{HO}-1$ is currently recognized as a potential antioxidant that may be effective in protecting against oxidative stress. ${ }^{40,41}$ To explore the cytoprotective effects of HO-1 against oxidative stress due to CuNP-induced damage, hemin was used to induce HO-1 overexpression in vitro. We found that the induction of HO-1 increased antioxidant defenses and decreased ROS generation. These results indicate that HO-1 may protect granulosa cells against oxidative stress triggered by CuNPs. Therefore, it can be speculated that high levels of HO-1 expression play a stress-protection role in this model of CuNP-induced damage.

Notably, this study only examined the cytoprotective effect of high levels of HO-1 expression. However, there may be other protective pathways in this CuNP-induced damage model that remain to be determined in future studies. Further investigation is also required to determine whether enhanced rescue measures can reverse oxidative stressinduced injury and the optimal timing of the intervention to maximize protection from oxidative stress-induced damage. 

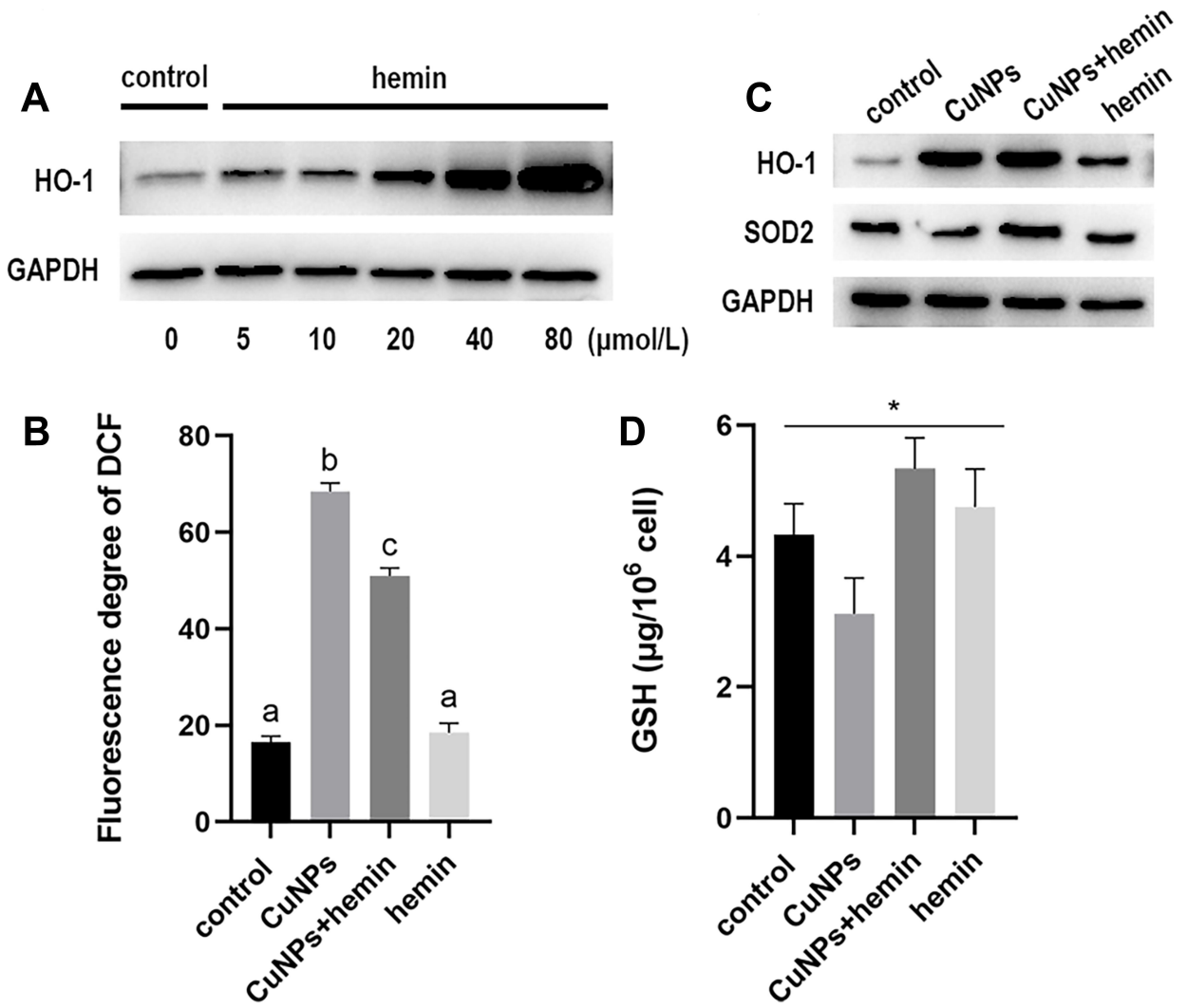

Figure 4 Effect of HO-I induction in COV434 cells exposed to CuNPs.

Notes: (A) Western blotting images of HO-I protein expression. COV434 cells were treated with different concentrations ( $\mu$ mol/L) of hemin for $48 \mathrm{~h}$. (B) ROS levels in COV434 cells exposed to CuNPs before and after HO-I overexpression. (C) Western blotting images of HO-I and SOD2 proteins. (D) GSH levels in COV434 cells exposed to CuNPs before and after HO-I overexpression. ${ }^{*} p<0.05 ;{ }^{a, b, c}$ Indicate statistical significance, with the same letters indicating no difference between groups. Abbreviations: HO-I, heme oxygenase I; CuNPs, copper nanoparticles; ROS, reactive oxygen species; SOD2, superoxide dismutase 2; GSH, glutathione peroxidase.

\section{Conclusion}

In summary, we have demonstrated that oxidative stress is one of the cytotoxic effects of CuNPs on ovarian granulosa cells, and that CuNPs may strongly induce HO-1 via the MAPK14-Nrf2 signaling pathway. The change of mitochondrial membrane potential, the increase of apoptosis rate and oxidative stress are the key mechanisms of cytotoxicity triggered by CuNPs, which can provide evidence for the study of CuNPs-induced damage. Moreover, HO-1 protects against CuNP-induced cytotoxicity by reducing oxidative stress. Therefore, the upregulation of HO-1 may help prevent and treat ovarian dysfunction caused by nanoparticles. We also realize the limitations of this study. Our results are only a preliminary exploration on the molecular mechanism and need to be further verified by in vivo models.

\section{Acknowledgments}

This work was supported by the National Natural Science Foundation of China (No. 81971435)

\section{Disclosure}

The authors report no conflicts of interest in this work.

\section{References}

1. Nel A, Xia T, Madler L, Li N. Toxic potential of materials at the nanolevel. Science. 2006;311(5761):622-627. doi:10.1126/ science. 1114397

2. Dong C, Feng W, Xu W, et al. The coppery age: copper $(\mathrm{Cu})$-involved nanotheranostics. Adv Sci (Weinh). 2020;7(21):2001549. doi:10.1002/ advs.202001549

3. Hassan S, Hassan FU, Rehman MS. Nano-particles of trace minerals in poultry nutrition: potential applications and future prospects. Biol Trace Elem Res. 2020;195(2):591-612. doi:10.1007/s12011-019$01862-9$

4. Deka P, Borah BJ, Saikia H, Bharali P. Cu-based nanoparticles as emerging environmental catalysts. Chem Rec. 2019;19(2-3):462-473. doi: $10.1002 /$ tcr. 201800055

5. Ameh T, Sayes CM. The potential exposure and hazards of copper nanoparticles: a review. Environ Toxicol Pharmacol. 2019;71:103220. doi:10.1016/j.etap.2019.103220

6. Anjum NA, Adam V, Kizek R, et al. Nanoscale copper in the soil-plant system - toxicity and underlying potential mechanisms. Environ Res. 2015;138:306-325. doi:10.1016/j.envres.2015.02.019

7. Chen Z, Meng H, Xing G, et al. Acute toxicological effects of copper nanoparticles in vivo. Toxicol Lett. 2006;163(2):109-120. doi:10.1016/ j.toxlet.2005.10.003 
8. Tang $\mathrm{H}, \mathrm{Xu} \mathrm{M}$, Zhou X, et al. Acute toxicity and biodistribution of different sized copper nano-particles in rats after oral administration. Mater Sci Eng C Mater Biol Appl. 2018;93:649-663. doi:10.1016/j. msec.2018.08.032

9. Lee IC, Ko JW, Park SH, et al. Comparative toxicity and biodistribution of copper nanoparticles and cupric ions in rats. Int J Nanomedicine. 2016;11:2883-2900. doi:10.2147/IJN.S106346

10. Bai R, Zhang L, Liu Y, et al. The dose-dependent toxicological effects and potential perturbation on the neurotransmitter secretion in brain following intranasal instillation of copper nanoparticles. Nanotoxicology. 2012;6(5):562-575. doi:10.3109/17435390.201 1.590906

11. Yang J, Hu S, Rao M, et al. Copper nanoparticle-induced ovarian injury, follicular atresia, apoptosis, and gene expression alterations in female rats. Int J Nanomedicine. 2017;12:5959-5971. doi:10.2147/ IJN.S139215

12. Sirotkin AV, Radosová M, Tarko A, Martín-García I, Alonso F. Effect of morphology and support of copper nanoparticles on basic ovarian granulosa cell functions. Nanotoxicology. 2020;14(5):683-695. doi:10.1080/17435390.2020.1736680

13. Roychoudhury S, Nath S, Massanyi P, Stawarz R, Kacaniova M, Kolesarova A. Copper-induced changes in reproductive functions: in vivo and in vitro effects. Physiol Res. 2016;65(1):11-22. doi: $10.33549 /$ physiolres. 933063

14. Midander K, Cronholm P, Karlsson HL, et al. Surface characteristics, copper release, and toxicity of nano- and micrometer-sized copper and copper(II) oxide particles: a cross-disciplinary study. Small. 2009;5(3):389-399. doi:10.1002/smll.200801220

15. Meng H, Chen Z, Xing G, et al. Ultrahigh reactivity provokes nanotoxicity: explanation of oral toxicity of nano-copper particles. Toxicol Lett. 2007;175(1-3):102-110. doi:10.1016/j.toxlet.20 07.09.015

16. Strauch BM, Niemand RK, Winkelbeiner NL, Hartwig A. Comparison between micro- and nanosized copper oxide and water soluble copper chloride: interrelationship between intracellular copper concentrations, oxidative stress and DNA damage response in human lung cells. Part Fibre Toxicol. 2017;14(1):28. doi:10.1186/ s12989-017-0209-1

17. Xu P, Xu J, Liu S, Yang Z. Nano copper induced apoptosis in podocytes via increasing oxidative stress. J Hazard Mater. 2012;241-242:279-286. doi:10.1016/j.jhazmat.2012.09.041

18. Denluck L, Wu F, Crandon LE, Harper BJ, Harper SL. Reactive oxygen species generation is likely a driver of copper based nanomaterial toxicity. Environ Sci Nano. 2018;5(6):1473-1481. doi:10.1039/C8EN00055G

19. Manna P, Ghosh M, Ghosh J, Das J, Sil PC. Contribution of nanocopper particles to in vivo liver dysfunction and cellular damage: role of I $\mathrm{B} \alpha / \mathrm{NF}-\kappa \mathrm{B}$, MAPKs and mitochondrial signal. Nanotoxicology. 2012;6(1):1-21. doi:10.3109/17435390.2011.552124

20. Sarkar A, Das J, Manna P, Sil PC. Nano-copper induces oxidative stress and apoptosis in kidney via both extrinsic and intrinsic pathways. Toxicology. 2011;290(2-3):208-217. doi:10.1016/j. tox.2011.09.086

21. Zhou X, Zhao L, Luo J, et al. The toxic effects and mechanisms of nano-cu on the spleen of rats. Int J Mol Sci. 2019;20(6):1469. doi:10.3390/ijms20061469

22. Da Clarke HJ. Regulation of germ cell development by intercellular signaling in the mammalian ovarian follicle. Wiley Interdiscip Rev Dev Biol. 2018;7(1):1. doi:10.1002/wdev.294

23. Lushchak VI. Free radicals, reactive oxygen species, oxidative stress and its classification. Chem Biol Interact. 2014;224:164-175. doi:10.1016/j.cbi.2014.10.016

24. Newsholme P, Cruzat VF, Keane KN, Carlessi R, de Bittencourt PI Jr. Molecular mechanisms of ROS production and oxidative stress in diabetes. Biochem J. 2016;473(24):4527-4550.
25. Nitti M, Piras S, Marinari UM, Moretta L, Pronzato MA, Furfaro AL. HO-1 induction in cancer progression: a matter of cell adaptation. Antioxidants (Basel). 2017;6(2):29. doi:10.3390/antiox6020029

26. Wang Y, Yang C, Elsheikh NAH, et al. HO-1 reduces heat stress-induced apoptosis in bovine granulosa cells by suppressing oxidative stress. Aging (Albany NY). 2019;11(15):5535-5547. doi:10.18632/aging.102136

27. Song C, Heping H, Shen Y, et al. AMPK/p38/Nrf2 activation as a protective feedback to restrain oxidative stress and inflammation in microglia stimulated with sodium fluoride. Chemosphere. 2020;244:125495. doi:10.1016/j.chemosphere.2019.125495

28. Campbell JH, Heikkila JJ. Effect of hemin, baicalein and heme oxygenase-1 (HO-1) enzyme activity inhibitors on Cd-induced accumulation of HO-1, HSPs and aggresome-like structures in Xenopus kidney epithelial cells. Comp Biochem Physiol C Toxicol Pharmacol. 2018;210:1-17. doi:10.1016/j.cbpc.2018.04.003

29. Zhang CH, Wang Y, Sun QQ, et al. Copper nanoparticles show obvious in vitro and in vivo reproductive toxicity via ERK mediated signaling pathway in female mice. Int J Biol Sci. 2018;14 (13):1834-1844. doi:10.7150/ijbs.27640

30. Dhupal M, Oh JM, Tripathy DR, Kim SK, Koh SB, Park KS. Immunotoxicity of titanium dioxide nanoparticles via simultaneous induction of apoptosis and multiple toll-like receptors signaling through ROS-dependent SAPK/JNK and p38 MAPK activation. Int J Nanomedicine. 2018;13:6735-6750. doi:10.2147/IJN.S176087

31. Moniczewski A, Gawlik M, Smaga I, et al. Oxidative stress as an etiological factor and a potential treatment target of psychiatric disorders. Part 1. Chemical aspects and biological sources of oxidative stress in the brain. Pharmacol Rep. 2015;67(3):560-568. doi:10.1016/j.pharep.2014.12.014

32. Cuadrado A, Nebreda AR. Mechanisms and functions of p 38 MAPK signalling. Biochem J. 2010;429(3):403-417. doi:10.1042/BJ20 100323

33. Ono K, Han J. The p38 signal transduction pathway: activation and function. Cell Signal. 2000;12(1):1-13. doi:10.1016/S0898-6568(99) 00071-6

34. Ahmad MI, Zou X, Ijaz MU, et al. Processed meat protein promoted inflammation and hepatic lipogenesis by upregulating Nrf2/Keap1 signaling pathway in Glrx-deficient mice. J Agric Food Chem. 2019;67(32):8794-8809. doi:10.1021/acs.jafc.9b03136

35. Aueviriyavit S, Phummiratch D, Maniratanachote R. Mechanistic study on the biological effects of silver and gold nanoparticles in Caco-2 cells-induction of the Nrf2/HO-1 pathway by high concentrations of silver nanoparticles. Toxicol Lett. 2014;224(1):73-83. doi:10.1016/j.toxlet.2013.09.020

36. Liu W, Hu T, Zhou L, et al. Nrf2 protects against oxidative stress induced by $\mathrm{SiO}_{2}$ nanoparticles. Nanomedicine (Lond). 2017;12 (19):2303-2318. doi:10.2217/nnm-2017-0046

37. Wang J, Li N, Zheng L, et al. P38-Nrf-2 signaling pathway of oxidative stress in mice caused by nanoparticulate $\mathrm{TiO}_{2}$. Biol Trace Elem Res. 2011;140(2):186-197. doi:10.1007/s12011-010-8687-0

38. Ko WC, Shieh JM, Wu WB. P38 MAPK and Nrf2 activation mediated naked gold nanoparticle induced heme oxygenase-1 expression in rat aortic vascular smooth muscle cells. Arch Med Res. 2020;51(5):388-396. doi:10.1016/j.arcmed.2020.04.015

39. Loboda A, Damulewicz M, Pyza E, Jozkowicz A, Dulak J. Role of Nrf2/HO-1 system in development, oxidative stress response and diseases: an evolutionarily conserved mechanism. Cell Mol Life Sci. 2016;73(17):3221-3247.

40. Chen S, Wang X, Nisar MF, Lin M, Zhong JL. Heme oxygenases: cellular multifunctional and protective molecules against UV-induced oxidative stress. Oxid Med Cell Longev. 2019;2019:5416728. doi: $10.1155 / 2019 / 5416728$

41. Liu X, Lin X, Zhang S, et al. Lycopene ameliorates oxidative stress in the aging chicken ovary via activation of $\mathrm{Nrf2} / \mathrm{HO}-1$ pathway. Aging (Albany NY). 2018;10(8):2016-2036. doi:10.18632/aging.101526 


\section{Publish your work in this journal}

The International Journal of Nanomedicine is an international, peerreviewed journal focusing on the application of nanotechnology in diagnostics, therapeutics, and drug delivery systems throughout the biomedical field. This journal is indexed on PubMed Central, MedLine, CAS, SciSearch ${ }^{\mathbb{B}}$, Current Contents ${ }^{\mathbb{B}} /$ Clinical Medicine,
Journal Citation Reports/Science Edition, EMBase, Scopus and the Elsevier Bibliographic databases. The manuscript management system is completely online and includes a very quick and fair peer-review system, which is all easy to use. Visit http://www.dovepress.com/ testimonials.php to read real quotes from published authors.

Submit your manuscript here: https://www.dovepress.com/international-journal-of-nanomedicine-journal 